$1-2-2017$

\title{
Reducing falls in a model of impaired cognitive control of movement (Commentary on Kucinski etal.)
}

Joshua A. Burk

College of William and Mary, jabur2@wm.edu

Follow this and additional works at: https://scholarworks.wm.edu/aspubs

\section{Recommended Citation}

Burk, Joshua A., Reducing falls in a model of impaired cognitive control of movement (Commentary on Kucinski etal.) (2017).

10.1111/ejn. 13466

This Other is brought to you for free and open access by the Arts and Sciences at W\&M ScholarWorks. It has been accepted for inclusion in Arts \& Sciences Articles by an authorized administrator of W\&M ScholarWorks. For more information, please contact scholarworks@wm.edu. 


\title{
COMMENTARY Reducing falls in a model of impaired cognitive control of movement (Commentary on Kucinski et al.)
}

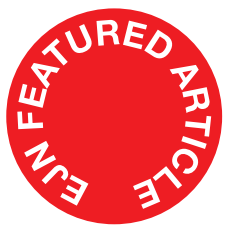

\author{
Joshua A. Burk \\ Department of Psychology, College of William \& Mary, Williamsburg, VA 23187, USA
}

Millions of individuals 65 years of age and older fall each year, leading to direct medical costs of approximately \$34 billion per year in the US alone (Stevens et al., 2006). Cognitive decline has often been used as an exclusion criterion in studies regarding falls, although changes in cognitive processing are now thought to increase the risk of falls (Domingos et al., 2015). Older adults tend to have more difficulty dividing attention among multiple tasks (Verhaeghen \& Cerella, 2002). Thus, challenges to the limits of attentional processing, such as distracting stimuli, may limit the ability to attend and make adjustments in order to maintain balance, thereby increasing the likelihood of falls. More recent experiments have focused on attentional deficits in conditions such as Parkinson's disease, associated with motor deficits and increased risk of falls (Pelosin et al., 2016). This mounting evidence leads to the conclusion that drug treatments that facilitate attentional processing may decrease the risk of falls.

A next step in this area is to identify relevant underlying mechanisms involved in motor control and attention, assess changes in the brain regions in relevant conditions such as Parkinson's disease and then evaluate whether treatments that facilitate attentional processing can decrease the rate of falls. Dopamine release within the dorsal striatum has long been associated with various aspects of motor control, including initiating and stopping movements, and degeneration of dopaminergic pathways from the substantia nigra to the striatum is well-established in Parkinson's disease. Several neurotransmitter systems have been implicated in attention, with strong evidence indicating that basal forebrain corticopetal cholinergic neurons are necessary for attention (Klinkenberg et al., 2011). Kucinski et al. (2013) established a model whereby lesions of dopaminergic terminals in the dorsal striatum along with lesions of basal forebrain corticopetal cholinergic neurons increased the rate of falls by rats in a motor task that varied demands to maintain balance and included presentation of distracting stimuli. Importantly, other studies in humans also provide evidence that dysfunctional cholinergic activity contributes to an increase in falls in patients with Parkinson's disease and in the elderly, supporting the translational potential of the animal model (Pelosin et al., 2016). The next step, described in the paper in this issue by Kucinski et al., is to test whether treatments that target depressed cortical cholinergic function attenuate the increased fall rate in this animal model.

Acetylcholinesterase (AChE) inhibitors, such as donepezil, have been used with some success to restore cholinergic deficiency and improve cognitive function in patients with Alzheimer's disease. However, given the limited benefits of AChE inhibitors, clearly other treatments are needed to improve disrupted cognitive processes, including attention. In the present manuscript, the effects of co-administering donepezil with the 5-HT6 receptor antagonist, idalopirdine, were tested in a task that assessed motor function. The task demands were increased by requiring more challenging movements and presenting distractors. Animals were also tested in a measure of visual sustained attention. Co-administration of donepezil and idalopirdine has shown beneficial effects in patients with Alzheimer's disease, potentially through pro-cholinergic effects (Herrik et al., 2016). The key finding in the present experiment is that co-administration of donepezil and idalopirdine reduced deficits in complex motor movements, particularly when distracters were presented, in animals with a reduced dopamine input to the dorsal striatum and reduced cortical cholinergic projections. Interestingly, the beneficial effects of drug treatment were largely due to improvement when the rats engaged in short stoppages of movements, presumably due to an impaired ability to adjust motor programming to maintain balance during challenging conditions. The sustained attention task deficits in the lesion model were not improved by drug treatments. The hypothesized neural mechanisms for these effects are that donepezil and idalpirdine treatment act downstream of the mechanisms involved in attentional control of movement or possibly upon brainstem cholinergic systems. A clear next step is the need to better understand the mechanisms by which this drug treatment improves performance in this test of motor function.

The present findings encourage research into whether other drugs that enhance attention have the potential to limit falls in conditions associated with dysfunctional striatal dopamine and cortical acetylcholine. For example stimulants such as caffeine, with relatively low abuse potential, may be useful to consider when testing whether attentional enhancement can decrease the likelihood of falls. The present findings also have implications beyond conditions in which motor function is impaired. Indeed, stimulants, such as amphetamine, which can enhance attention, have been used to improve normal motor performance, notably athletic performance (Tokish et al., 2004). It remains to be established whether, in humans with intact striatal dopamine and cortical cholinergic systems, drugs such as donepezil and idalopirdine can enhance motor abilities. In summary, the present results offer an encouraging potential treatment to address a major health issue, by targeting attentional processing in order to decrease the likelihood of falls associated with reduced cortical cholinergic and striatal dopaminergic innervation. 


\section{Conflict of interests}

The author declares no conflict of interest associated with this manuscript.

\section{References}

Domingos, J.M., Godinho, C., Dean, J., Coelho, M., Pinto, A., Bloem, B.R. \& Ferreira, J.J. (2015) Cognitive impairment in fall-related studies in Parkinson's disease. J. Parkinsons Dis., 5, 453-469.

Herrik, K.F., Mork, A., Richard, N., Bundgaard, C., Baslund, J.F. \& de Jong, I.E. (2016) The 5-HT receptor antagonist idalopirdine potentiates the effects of acetylcholinesterase inhibition on neuronal network oscillations and extracellular acetylcholine levels in the rat dorsal hippocampus. Neuropharmacology, 107, 351-363.

Klinkenberg, I., Sambeth, A. \& Blokland, A. (2011) Acetylcholine and attention. Behav. Brain Res., 221, 430-442.

Kucinski, A., Paolone, G., Bradshaw, M., Albin, R. \& Sarter, M. (2013) Modeling fall propensity in Parkinson's disease: deficits in attentional control of complex movements in rats with cortical-cholinergic and striatal-dopaminergic deafferentation. J. Neurosci., 33, 16522-16539.

Kucinski, A., de Jong, I.E.M. \& Sarter, M. (2016) Reducing falls in Parkinson's disease: interactions between donepezil and the 5-HT6 receptor antagonist idalopirdine on falls in a rat model of impaired cognitive control of complex movements. Eur. J. Neurosci., in press.

Pelosin, E., Ogliastro, C., Lagravinese, G., Bonassi, G., Mirelman, A., Hausdorff, J.M., Abbruzzese, G. \& Avanzino, L. (2016) Attentional control of gait and falls: is cholinergic dysfunction a common substrate in the elderly and Parkinson's disease? Front. Aging Neurosci., 8, 104.

Stevens, J.A., Corso, P.S., Finkelstein, E.A. \& Miller, T.R. (2006) The costs of fatal and nonfatal falls among older adults. Inj. Prev., 12, $290-295$.

Tokish, J.M., Kocher, M.S. \& Hawkins, R.J. (2004) Ergogenic aids: a review of basic science, performance, side effects, and status in sports. Am. J. Sport. Med., 32, 1543-1553.

Verhaeghen, P. \& Cerella, J. (2002) Aging, executive control, and attention: a review of meta-analyses. Neurosci. Biobehav. R., $26,849-857$. 